\title{
Formulation and experimental validation of space-fractional Timoshenko beam model with functionally graded materials effects
}

\author{
Paulina Stempin ${ }^{1} \cdot$ Wojciech Sumelka ${ }^{1}$
}

Received: 20 October 2020 / Accepted: 30 January 2021 / Published online: 13 March 2021

(c) The Author(s) 2021

\begin{abstract}
In this study, the static bending behaviour of a size-dependent thick beam is considered including FGM (Functionally Graded Materials) effects. The presented theory is a further development and extension of the space-fractional (non-local) EulerBernoulli beam model (s-FEBB) to space-fractional Timoshenko beam (s-FTB) one by proper taking into account shear deformation. Furthermore, a detailed parametric study on the influence of length scale and order of fractional continua for different boundary conditions demonstrates, how the non-locality affects the static bending response of the s-FTB model. The differences in results between s-FTB and s-FEBB models are shown as well to indicate when shear deformations need to be considered. Finally, material parameter identification and validation based on the bending of SU-8 polymer microbeams confirm the effectiveness of the presented model.
\end{abstract}

Keywords Timoshenko beam $\cdot$ Non-local model $\cdot$ Fractional calculus $\cdot$ Microbeam bending

$\begin{array}{ll}\text { Abbreviations } & \\ \text { FGM } & \text { Functionally graded materials } \\ \text { s-FEBB } & \text { Space-fractional Euler-Bernoulli beam } \\ & \text { model } \\ \text { S-FTB } & \text { Space-fractional Timoshenko beam } \\ \text { SE } & \text { Scale effect } \\ \text { RVE } & \text { Representative volume element } \\ \text { BD } & \text { Body dimensions } \\ \text { LS } & \text { Length scale } \\ \text { FDO } & \text { Fractional differential operator } \\ \text { CTB } & \text { Classical Timoshenko beam }\end{array}$

\section{Introduction}

The first works on scale effect (SE), which manifest dependence on the answer of the system (e.g. deformation of a material body - the crux of this paper) in relation to its dimensions, date to the investigations of Leonardo da Vinci

In Honor of Professor Tomasz Łodygowski on the Occasion of His 70th Birthday.

Wojciech Sumelka

wojciech.sumelka@put.poznan.pl

1 Institute of Structural Analysis, Poznan University of Technology, Piotrowo 5 Street, 60-965 Poznan, Poland at the very beginning of XVI century [1,2]. Since that time both experimental techniques [3-7] and theoretical concepts [8-13] for SE analysis were considerably developed and moreover SE phenomena were utilised successfully in the modern industry [14-18]. The main message is that from the standpoint of mechanics, which constitutes the central point of presented considerations, one can say that each structure, due to the complexity of material over different scales of observation, reveals SE. On the other hand, it is clear that the strength of SE phenomena is different depending on the analysed case and proportional to the ratio of the representative volume element (RVE) of a specific material to body dimensions (BD).

From the theoretical side, when constructing a mathematical model for the description of mechanical phenomena, one should choose certain mathematical objects proper to the experimental observation scale [19-22]. Herein, we operate on meso/macro level, therefore for SE modelling a phenomenological approach is used, thus in consequence RVE to $\mathrm{BD}$ ratio is mapped utilising so-called length scale (LS) parameter (it is clear that LS meaning is different depending on certain theory [15,23-26]). To be precise, the developed s-FTB theory is defined in the framework of space-Fractional Continuum Mechanics (s-FCM) [27,28], where LS is introduced through the fractional differential operator (FDO) and furthermore, an additional parameter which controls SE is 
introduced, namely order of FDO. Finally, because of the complex nature of mechanical properties through the beam thickness FGM concept is also used [29-32].

This paper is a continuation of previous studies focused on the development of non-local beam models [27,28]. The currently formulated space-Fractional Timoshenko beam model, compared to the space-Fractional Euler-Bernoulli beam model, takes into account the shear effect to extend modelling range for thick beams. The definition of rotation has been therefore extended by additional rotation resulting from non-local shear deformations. Hence, the appropriate governing equations and enriched numerical algorithm are provided. The discussion also includes a study of the influence of non-locality parameters for different boundary conditions on the static beam response to bending, a comparison study between s-FTB and s-FEBB models, and identification and validation of s-FTB model parameters for the experimental data of the SU-8 polymer microcantilever bending test (including FGM effects).

The paper is structured as follows. Section 2 deals with the s-FTB definition. Section 3 is devoted to the numerical scheme and parametric study. Section 4 provides experimental validation and finally Sect. 5 concludes the paper.

\section{Theory}

The space-Fractional Timoshenko beam (s-FTB) theory is an extension of the space-Fractional Euler-Bernoulli beam (sFEBB) theory [28] to include shear deformation and make it suitable for thick beams as mentioned in the introduction. As in the previous study, the non-locality is taken into account, based on the fractional elasticity concept $[33,34]$, by the following definition of small strain

$\stackrel{\diamond}{\varepsilon}_{i j}=\frac{1}{2} \ell_{f}^{\alpha-1}\left(\stackrel{\alpha}{\underset{x_{j}}{D}} u_{i}(\mathbf{x})+\stackrel{\alpha}{\underset{x_{i}}{D}} u_{j}(\mathbf{x})\right)$,

where $u_{i}$ are the components of the displacement vector, $\mathbf{x}$ is a spatial variable, whereas the term $\stackrel{\alpha}{D}($. $)$ denotes the Riesz-Caputo fractional derivative [35],

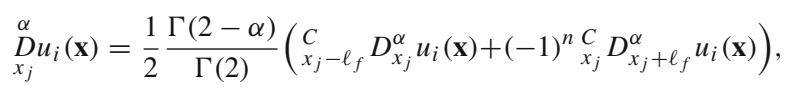

with the left-side and right-side Caputo derivatives

$$
\begin{aligned}
& { }_{x_{j}-\ell_{f}}^{C} D_{x_{j}}^{\alpha} u_{i}(\mathbf{x})=\frac{1}{\Gamma(n-\alpha)} \int_{x_{j}-\ell_{f}}^{x_{j}} \frac{u_{i}^{(n)}(\tau)}{\left(x_{j}-\tau\right)^{\alpha-n+1}} \mathrm{~d} \tau, \\
& { }_{x_{j}}^{C} D_{x_{j}+\ell_{f}}^{\alpha} u_{i}(\mathbf{x})=\frac{-1}{\Gamma(n-\alpha)} \int_{x_{j}}^{x_{j}+\ell_{f}} \frac{u_{i}^{(n)}(\tau)}{\left(x_{j}-\tau\right)^{\alpha-n+1}} \mathrm{~d} \tau,
\end{aligned}
$$

where $\Gamma$ is an Euler gamma function, $n=[\alpha]+1$, and [.] denotes the integer part of a real number, $\alpha \in(0,1]$ is the order of FDO, and $\ell_{f}$ is LS i.e. the surrounding affecting the considered material point. The concept of variable length scale [36] $\ell_{f}=\ell_{f}(\mathbf{x})$, as function decreasing at the boundaries, has been kept. These two parameters $\alpha$ and $\ell_{f}$ are regarded as associated with microstructure [37] and responsible for SE mapping.

In the presented study one considers the static bending behavior in the $x_{1} x_{3}$-plane, therefore, keeping the assumption that the cross-section is infinitely rigid in its own plane and remains plane after deformation. In consequence, the displacement field takes the form

$u_{1}\left(x_{1}, x_{2}, x_{3}\right)=x_{3} \Phi_{2}, \quad u_{2}\left(x_{1}, x_{2}, x_{3}\right)=0, \quad u_{3}\left(x_{1}, x_{3}, x_{3}\right)=\bar{u}_{3}$.

where $\bar{u}_{3}=\bar{u}_{3}\left(x_{1}\right)$ is the rigid body translation of the crosssection in 3-rd axis direction of the coordinate system and $\Phi_{2}=\Phi_{2}\left(x_{1}\right)$ is the rigid body rotation (positive keeping the right-hand rule). Rotation $\Phi_{2}$ depends on the Riesz-Caputo fractional derivative with the proportionality factor $\ell_{f}^{\alpha-1}$ and, by comparison to the already developed s-FEBB [28], is extended by an additional rotation due to the fractional shear deformation $\hat{\gamma}_{13}$,

$\Phi_{2}=-\ell_{f}^{\alpha-1}{\stackrel{\alpha}{D} \bar{u}_{1}}_{3}+\stackrel{\diamond}{\gamma}_{13}$

Herein, it should be emphasised that on one hand side the assumption Eq. (6) reflects the influence of microstructure on beam cross section rotation, but simultaneously acts as a consistency condition. Namely, it allows to obtain proper relation between the component of the fractional Cauchy strain $\stackrel{\diamond}{\varepsilon}_{13}$ and the fractional shear deformation $\grave{\gamma}_{13}$. The last statement causes that in limit case when s-FTB reduces to s-FEBB $\stackrel{\diamond}{\varepsilon}_{13}=0$ which is fundamental for s-FEBB [28].

Next, using Eqs (1) and (6) the nonzero fractional Cauchy strains are

$$
\begin{aligned}
& \stackrel{\diamond}{\varepsilon}_{11}=x_{3} \ell_{f}^{\alpha-1}\left[-\stackrel{\alpha}{D}\left(\ell_{f}^{\alpha-1}{\stackrel{\alpha}{D} \bar{u}_{3}}_{1}\right)+\stackrel{\alpha}{D}{\stackrel{\diamond}{\gamma_{1}}}_{13}\right], \\
& \stackrel{\diamond}{\varepsilon}_{13}=\stackrel{\diamond}{\varepsilon}_{31}=\frac{1}{2}\left[\left(-\ell_{f}^{\alpha-1} \stackrel{\alpha}{D} \bar{u}_{3}+\stackrel{\diamond}{\gamma}_{13}\right) \stackrel{\alpha}{D} x_{3}+\stackrel{\alpha}{D} \bar{u}_{3}\right] \ell_{f}^{\alpha-1}=\frac{1}{2} \stackrel{\diamond}{\gamma}_{13} .
\end{aligned}
$$

It is so because in Eq. $\left(7_{2}\right) \ell_{f}^{\alpha-1}{\stackrel{\alpha}{D} x_{3}}_{3}=1$, then $\stackrel{\diamond}{\varepsilon}_{13}=\frac{1}{2} \stackrel{\diamond}{\gamma}_{13}$. The corresponding stresses are

$$
\begin{aligned}
\sigma_{11} & =x_{3} \ell_{f}^{\alpha-1}\left[-\stackrel{\alpha}{\underset{x}{D}}\left(\ell_{f}^{\alpha-1} \stackrel{\alpha}{D} \bar{u}_{3}\right)+\stackrel{\alpha}{D} \stackrel{\diamond}{\gamma}_{x_{1}}\right] E\left(x_{2}, x_{3}\right), \\
\sigma_{13} & =G \stackrel{\diamond}{\varepsilon}_{13},
\end{aligned}
$$

where $E\left(x_{2}, x_{3}\right)$ is Young's modulus, $G=G\left(x_{2}, x_{3}\right)=$ $\frac{E\left(x_{2}, x_{3}\right)}{2(1+v)}$ is Kirchhoff modulus and $v$ is Poisson's ratio. Based 
on the above results, the bending moment $M_{2}$ and the shear force $V_{3}$ can be expressed as

$M_{2}=M_{2}\left(x_{1}\right)=\int_{A} x_{3} \sigma_{11} \mathrm{~d} A$ and $V_{3}=V_{3}\left(x_{1}\right)=\int_{A} \sigma_{13} \mathrm{~d} A$.

and, by introducing Eq. (8), as

$$
\begin{aligned}
M_{2} & =\int_{A} x_{3} \sigma_{11} \mathrm{~d} A=\ell_{f}^{\alpha-1}\left[-\stackrel{\alpha}{D}\left(\ell_{f}^{\alpha-1} \underset{x_{1}}{D} \bar{u}_{3}\right)+\stackrel{\alpha}{D} \grave{\gamma}_{13}\right](E I)^{*}, \\
V_{3} & =\int_{A} \sigma_{13} \mathrm{~d} A=k(G A)^{*} \stackrel{\diamond}{\gamma}_{13},
\end{aligned}
$$

where $k$ is the shear correction factor, $(E I)^{*}=\int_{A} E\left(x_{2}, x_{3}\right)$ $x_{3}^{2} \mathrm{~d} A$ and $(G A)^{*}=\int_{A} G\left(x_{2}, x_{3}\right) \mathrm{d} A$. The relationship among $V_{3}, M_{2}$ and distributed load $p_{3}=p_{3}\left(x_{1}\right)$ (for the derivation of following formulas from the principle of virtual work see Appendix and [28]) is

$$
\begin{aligned}
& V_{3}=\underset{x_{1}}{D}\left(M_{2} \ell_{f}^{\alpha-1}\right), \\
& p_{3}=-\stackrel{\alpha}{D}\left(V_{x_{1}} \ell_{f}^{\alpha-1}\right) .
\end{aligned}
$$

Moreover, the shear force $V_{3}$, by using Eqs. (10) and (12), can be also expressed as

$$
V_{3}=\stackrel{\alpha}{D}\left\{\ell_{x_{1}}^{2 \alpha-2}\left[-\stackrel{\alpha}{D}\left(\ell_{x_{1}}^{\alpha-1}{\stackrel{\alpha}{D} \bar{u}_{3}}_{3}\right)+\stackrel{\alpha}{D} \grave{\gamma}_{13}\right]\right\}(E I)^{*}
$$

Finally, substituting Eq. (14) in Eq. (13), and comparing Eq. (14) and Eq. (11) we obtain the fractional equations governing the bending of s-FTB
Moreover, for $\alpha=1$, the rotations Eqs. (6) and (16) take the classical form for Timoshenko and Euler-Bernoulli beam theories, respectively

$\Phi_{2}=-\frac{\mathrm{d} \bar{u}_{3}}{\mathrm{~d} x_{1}}+\gamma_{13}$ and $\Phi_{2}=-\frac{\mathrm{d} \bar{u}_{3}}{\mathrm{~d} x_{1}}$,

and the governing equations Eqs. (15) 1 and (17) also reduce to the classical local descriptions

$\frac{\mathrm{d}^{4} \bar{u}_{3}}{\mathrm{~d} x_{1}^{4}}(E I)^{*}=p_{3}+\frac{\mathrm{d}^{3} \gamma_{13}}{\mathrm{~d} x_{1}^{3}}(E I)^{*}$ and $\frac{\mathrm{d}^{4} \bar{u}_{3}}{\mathrm{~d} x_{1}^{4}}(E I)^{*}=p_{3}$,

Timoshenko and Euler-Bernoulli beam theories, respectively.

\section{Numerical study}

\subsection{Discretization}

Equation (15) has been solved utilising the numerical method [38]. The beam was discretized in $n$ intervals of length $h$ (see Fig. 1). The trapezoidal rule [38-40] was applied to

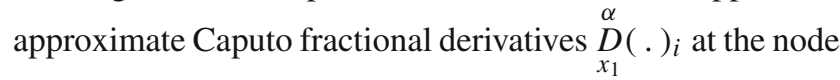

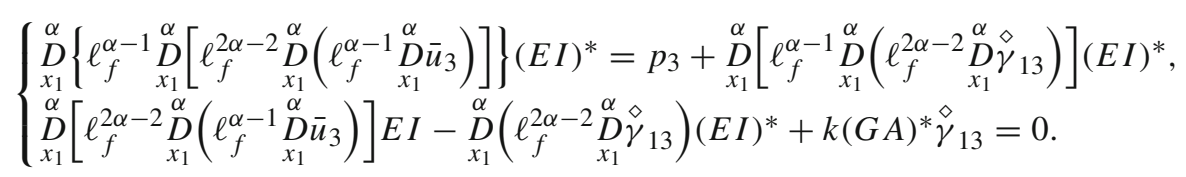

It is worth noting that when the shear deformation is neglected, i.e. $\vec{\gamma}_{13} \approx 0$, the rotation Eq. (6) takes the form

$\Phi_{2}=-\ell_{f}^{\alpha-1} \stackrel{\alpha}{D_{1}} \bar{u}_{3}$,

and consequently, Eq. (15) reduces to equation governing the bending of a s-FEBB model [28]

$$
\stackrel{\alpha}{D}\left\{\ell_{x_{1}}^{\alpha-1} \stackrel{\alpha}{\underset{x_{1}}{D}}\left[\ell_{f}^{2 \alpha-2} \underset{x_{1}}{D}\left(\ell_{f}^{\alpha-1} \underset{x_{1}}{\operatorname{D}} \bar{u}_{3}\right)\right]\right\}(E I)^{*}=p_{3}
$$

$x_{1}^{i}$ by the sum of first-order derivatives at nodes $x_{1}^{i-m} \div x_{1}^{i+m}$ (see the Fig. 1) with appropriate weight coefficients marked as $B, C^{a}$ and $C^{b}$, according to the Eq. (20) 


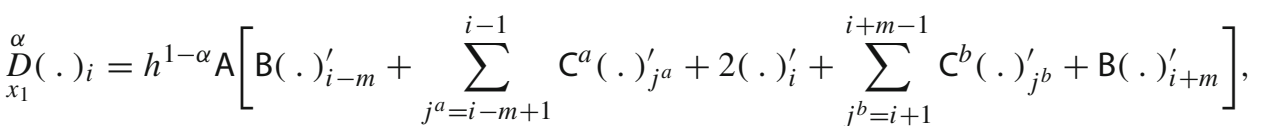

where

$$
\begin{gathered}
m=m_{i}=\left(\ell_{f}\right)_{i} / h \geq 2, \quad \mathrm{~A}=\frac{\Gamma(2-\alpha)}{2 \Gamma(2) \Gamma(3-\alpha)}, \quad \mathrm{B}=(m-1)^{2-\alpha}-(m+\alpha-2) m^{1-\alpha}, \\
\quad C^{a}=\left(i-j^{a}+1\right)^{2-\alpha}-2\left(i-j^{a}\right)^{2-\alpha}+\left(i-j^{a}-1\right)^{2-\alpha}, \\
\mathrm{C}^{b}=\left(j^{b}-i+1\right)^{2-\alpha}-2\left(j^{b}-i\right)^{2-\alpha}+\left(j^{b}-i-1\right)^{2-\alpha},
\end{gathered}
$$

and $\left(\ell_{f}\right)_{i}=\ell_{f}\left(x_{1}^{i}\right)$

Based on the Eq. (20), the numerical representation of the distributed load Eq. (15), shear force Eq. (14), bending moment Eq. (10) and rotation Eq. (6) at node $x_{1}^{i}$ are given by

$$
\begin{gathered}
\mathcal{D}_{i}(E I)^{*}=p_{3}\left(x_{1}^{i}\right)+\mathscr{C}_{i}(E I)^{*}, \quad \mathcal{C}_{i}(E I)^{*}-\mathscr{B}_{i}(E I)^{*}+k(G A)^{*} \hat{\gamma}_{13}\left(x_{1}^{i}\right)=0, \\
V_{3}\left(x_{1}^{i}\right)=\left(-\mathcal{C}_{i}+\mathscr{B}_{i}\right)(E I)^{*}, \text { or } V_{3}\left(x_{1}^{i}\right)=k(G A)^{*} \hat{\gamma}_{13}\left(x_{1}^{i}\right), \\
M_{2}\left(x_{1}^{i}\right)=\left(\ell_{f}^{\alpha-1}\right)_{i}\left(-\mathcal{B}_{i}+\mathscr{A}_{i}\right)(E I)^{*}, \quad \Phi_{2}\left(x_{1}^{i}\right)=-\left(\ell_{f}^{\alpha-1}\right)_{i} \mathcal{A}_{i}+\hat{\gamma}_{13}\left(x_{1}^{i}\right),
\end{gathered}
$$

where

$$
\begin{aligned}
& \mathcal{D}_{i}=\stackrel{\alpha}{D}\left\{\left(\ell_{x_{1}}^{\alpha-1}\right)_{i} \underset{x_{1}}{\stackrel{\alpha}{D}}\left[\left(\ell_{f}^{2 \alpha-2}\right)_{i} \underset{x_{1}}{\stackrel{\alpha}{D}}\left(\left(\ell_{f}^{\alpha-1}\right)_{i} \underset{x_{1}}{\stackrel{\alpha}{u_{3}} \bar{u}_{3}}\left(x_{1}^{i}\right)\right)\right]\right\}=\underset{x_{1}}{\stackrel{\alpha}{D}}\left[\left(\ell_{f}^{\alpha-1} \mathcal{C}\right)_{i}\right]=h^{1-\alpha} \mathrm{A}\left[\mathrm{B}\left(\ell_{f}^{\alpha-1} \mathcal{C}\right)_{i-m}^{\prime}\right. \\
& \left.+\sum_{j^{a}=i-m+1}^{i-1} \mathrm{C}^{a}\left(\ell_{f}^{\alpha-1} \mathcal{C}\right)_{j^{a}}^{\prime}+2\left(\ell_{f}^{\alpha-1} \mathcal{C}\right)_{i}^{\prime}+\sum_{j^{b}=i+1}^{i+m-1} \mathrm{C}^{b}\left(\ell_{f}^{\alpha-1} \mathcal{C}\right)_{j^{b}}^{\prime}+\mathrm{B} \cdot\left(\ell_{f}^{\alpha-1} \mathcal{C}\right)_{i+m}^{\prime}\right], \\
& \mathcal{C}_{i}=\stackrel{\alpha}{\underset{x_{1}}{D}}\left[\left(\ell_{f}^{2 \alpha-2}\right)_{i} \underset{x_{1}}{\stackrel{\alpha}{D}}\left(\left(\ell_{f}^{\alpha-1}\right)_{i} \underset{x_{1}}{\stackrel{\alpha}{D} \bar{u}_{3}}\left(x_{1}^{i}\right)\right)\right]=\stackrel{\alpha}{\underset{x_{1}}{D}}\left[\left(\ell_{f}^{2 \alpha-2} \mathcal{B}\right)_{i}\right]=h^{1-\alpha} \mathrm{A}\left[\mathrm{B}\left(\ell_{f}^{2 \alpha-2} \mathcal{B}\right)_{i-m}^{\prime}\right. \\
& \left.+\sum_{j^{a}=i-m+1}^{i-1} \mathrm{C}^{a}\left(\ell_{f}^{2 \alpha-2} \mathcal{B}\right)_{j^{a}}^{\prime}+2\left(\ell_{f}^{2 \alpha-2} \mathcal{B}\right)_{i}^{\prime}+\sum_{j^{b}=i+1}^{i+m-1} \mathrm{C}^{b}\left(\ell_{f}^{2 \alpha-2} \mathcal{B}\right)_{j^{b}}^{\prime}+\mathrm{B}\left(\ell_{f}^{2 \alpha-2} \mathcal{B}\right)_{i+m}^{\prime}\right] \text {, } \\
& \mathcal{B}_{i}=\stackrel{\alpha}{D}\left[\left(\ell_{x_{1}}^{\alpha-1}\right)_{i} \underset{x_{1}}{\stackrel{\alpha}{D} \bar{u}_{3}}\left(x_{1}^{i}\right)\right]=\stackrel{\alpha}{D}\left[\left(\ell_{x_{1}}^{\alpha-1} \mathcal{A}\right)_{i}\right]=h^{1-\alpha} \mathrm{A}\left[\mathrm{B}\left(\ell_{f}^{\alpha-1} \mathcal{A}\right)_{i-m}^{\prime}\right. \\
& \left.+\sum_{j^{a}=i-m+1}^{i-1} \mathrm{C}^{a}\left(\ell_{f}^{\alpha-1} \mathcal{A}\right)_{j^{a}}^{\prime}+2\left(\ell_{f}^{\alpha-1} \mathcal{A}\right)_{i}^{\prime}+\sum_{j^{b}=i+1}^{i+m-1} \mathrm{C}^{b}\left(\ell_{f}^{\alpha-1} \mathcal{A}\right)_{j^{b}}^{\prime}+\mathrm{B}\left(\ell_{f}^{\alpha-1} \mathcal{A}\right)_{i+m}^{\prime}\right],
\end{aligned}
$$

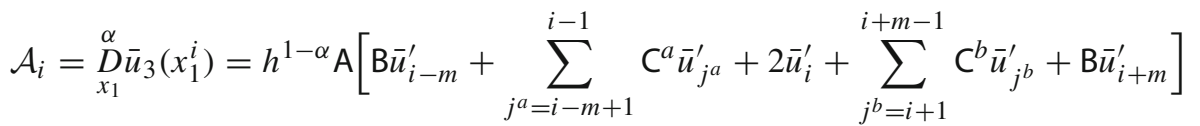

$$
\begin{aligned}
& \mathscr{C}_{i}=\stackrel{\alpha}{D}\left[\left(\ell_{f}^{\alpha-1}\right)_{i} \underset{x_{1}}{\stackrel{\alpha}{D}}\left(\left(\ell_{f}^{2 \alpha-2}\right)_{i} \underset{x_{1}}{\stackrel{\alpha}{\stackrel{\gamma}{\gamma}}}{ }_{13}\left(x_{1}^{i}\right)\right)\right]=\stackrel{\alpha}{\underset{x_{1}}{D}}\left[\left(\ell_{f}^{\alpha-1} \mathscr{B}\right)_{i}\right]=h^{1-\alpha} \mathrm{A}\left[\mathrm{B}\left(\ell_{f}^{\alpha-1} \mathscr{B}\right)_{i-m}^{\prime}\right. \\
& \left.+\sum_{j^{a}=i-m+1}^{i-1} \mathrm{C}^{a}\left(\ell_{f}^{\alpha-1} \mathscr{B}\right)_{j^{a}}^{\prime}+2\left(\ell_{f}^{\alpha-1} \mathscr{B}\right)_{i}^{\prime}+\sum_{j^{b}=i+1}^{i+m-1} \mathrm{C}^{b}\left(\ell_{f}^{\alpha-1} \mathscr{B}\right)_{j^{b}}^{\prime}+\mathrm{B}\left(\ell_{f}^{\alpha-1} \mathscr{B}\right)_{i+m}^{\prime}\right],
\end{aligned}
$$


Fig. 1 Discretization of the analysed beam of length $L-$ homogeneous grid: real nodes $x_{1}^{0} \div x_{1}^{n}$; fictitious nodes $x_{1}^{-8} \div x_{1}^{-1}$ and $x_{1}^{n+1} \div x_{1}^{n+8}$

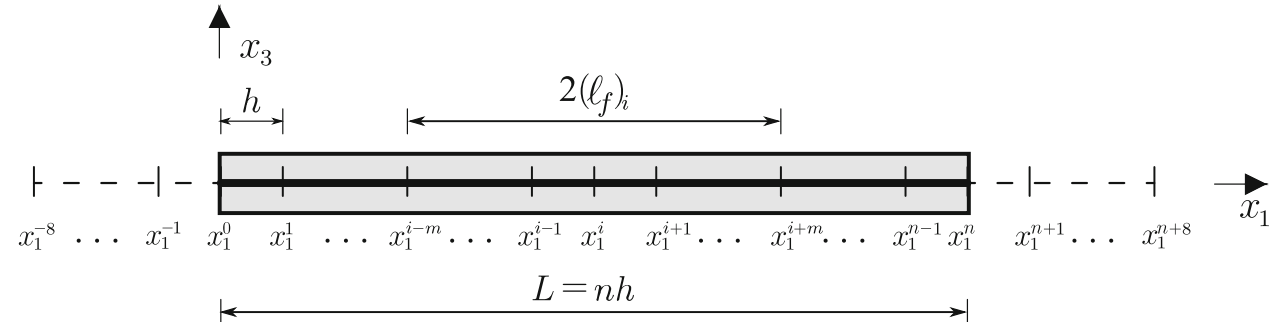

It should be pointed out that the first derivatives ( . $)^{\prime}$ in Eqs. $(23 \div 29)$ are approximated by forward, backward or central difference formulae at the relevant nodes according to Eq. (30) and Table 1,

$(.)_{i}^{\prime}=\left[-(.)_{i+N_{1}-N_{2}}+(.)_{i+N_{1}+N_{2}}\right] \frac{1}{2 N_{2} h}$,

where $N_{1}$ and $N_{2}$ determine which finite difference scheme has been applied (see Table 1).

One should conclude that similarly to Eq. (19), for $\alpha=1$ Eq. (22) returns for $N_{1}=0$ and $N_{2}=\frac{1}{2}$ to the classical central difference scheme $\left(\mathrm{A}=\frac{1}{2}, \mathrm{~B}=\mathrm{C}^{a}=\mathrm{C}^{b}=0\right)$ where $\Phi_{i}=\Phi_{2}\left(x_{1}^{i}\right), M_{i}=M_{2}\left(x_{1}^{i}\right), V_{i}=V_{3}\left(x_{1}^{i}\right), p_{i}=$ $p_{3}\left(x_{1}^{i}\right), \gamma_{i}=\gamma_{13}\left(x_{1}^{i}\right)$ and $u_{i}=\bar{u}_{3}\left(x_{1}^{i}\right)$.

Applied numerical methods have resulted in fictitious nodes $\left(x_{1}^{-8} \div x_{1}^{-1}\right.$ and $\left.x_{1}^{n+1} \div x_{1}^{n+8}\right)$ outside the beam in addition to real nodes $\left(x_{1}^{0} \div x_{1}^{n}\right)$ - see Fig. 1 . The application of the variable length scale $\ell_{f}(x)$, which is decreasing at the boundaries [36], results in only 8 fictitious nodes $\left(x_{1}^{-8} \div x_{1}^{-1}\right.$, $x_{1}^{n+1} \div x_{1}^{n+8}$ ) on each side of the beam. These points are eliminated in final set of equations, by the analogy to the approach presented in [36], by equating of the finite difference approximation

$$
\begin{aligned}
\Phi_{i} & =-\frac{-u_{i-1 / 2}+u_{i+1 / 2}}{h}+\gamma_{i}, \\
M_{i} & =\left(-\frac{u_{i-1}-2 u_{i}+u_{i+1}}{h^{2}}+\frac{-\gamma_{i-1 / 2}+\gamma_{i+1 / 2}}{h}\right)(E I)^{*}, \\
V_{i} & =\left(-\frac{-u_{i-3 / 2}+3 u_{i-1 / 2}-3 u_{i+1 / 2}+u_{i+3 / 2}}{h^{3}}+\frac{\gamma_{i-1}-2 \gamma_{i}+\gamma_{i+1}}{h^{2}}\right)(E I)^{*},
\end{aligned}
$$

and

$$
\frac{u_{i-2}-4 u_{i-1}+6 u_{i}-4 u_{i+1}+u_{i+2}}{h^{4}}(E I)^{*}=p_{i}+\frac{-\gamma_{i-3 / 2}+3 \gamma_{i-1 / 2}-3 \gamma_{i+1 / 2}+\gamma_{i+3 / 2}}{h^{3}}(E I)^{*},
$$


Table 1 The applied finite difference schemes evaluated at node $x_{1}^{i}$ where $N_{1}$ and $N_{2}$ determine which finite difference scheme has been applied (see also Eq. (30) and Fig. 1)

\begin{tabular}{lllll}
\hline & Forward & Backward & Central & Central \\
& $N_{1}=\frac{1}{2}$, & $N_{1}=-\frac{1}{2}$, & $N_{1}=0$, & $N_{1}=0$, \\
& $N_{2}=\frac{1}{2}$ & $N_{2}=\frac{1}{2}$ & $N_{2}=\frac{1}{2}$ & $N_{2}=1$ \\
\hline$u_{i}^{\prime}$ & $x_{1}^{i}=x_{1}^{-8}$ & $x_{1}^{i}=x_{1}^{n+8}$ & $x_{1}^{i}=x_{1}^{-7.5} \div x_{1}^{n+7.5}$ & $x_{1}^{i}=x_{1}^{-7} \div x_{1}^{2} ; x_{1}^{n-2} \div x_{1}^{n+7}$ \\
$\left(\ell_{f}^{\alpha-1} \mathcal{A}\right)_{i}^{\prime}$ & $x_{1}^{i}=x_{1}^{-6}$ & $x_{1}^{i}=x_{1}^{n+6}$ & $x_{1}^{i}=x_{1}^{-5} \div x_{1}^{n+5}$ & \\
$\left(\ell_{f}^{2 \alpha-2} \mathcal{B}\right)_{i}^{\prime}$ & ${ }_{1}=x_{1}^{-4}$ & $x_{1}^{i}=x_{1}^{n+4}$ & $x_{1}^{i}=x_{1}^{-3.5} \div x_{1}^{n+3.5}$ & $x_{1}^{i}=x_{1}^{-3} \div x_{1}^{n+3}$ \\
$\left(\ell_{f}^{\alpha-1} \mathcal{C}\right)_{i}^{\prime}$ & $x_{1}^{i}=x_{1}^{-2}$ & $x_{1}^{i}=x_{1}^{n+2}$ & $x_{1}^{i}=x_{1}^{-1} \div x_{1}^{n+1}$ & \\
$\stackrel{\diamond}{\gamma}_{i}^{\prime}$ & $x_{1}^{i}=x_{1}^{-6}$ & $x_{1}^{i}=x_{1}^{n+6}$ & $x_{1}^{i}=x_{1}^{-5.5} \div x_{1}^{n+5.5}$ & $x_{1}^{i}=x_{1}^{-5} \div x_{1}^{2} ; x_{1}^{n-2} \div x_{1}^{n+5}$ \\
$\left(\ell_{f}^{2 \alpha-2} \mathscr{A}\right)_{i}^{\prime}$ & $x_{1}^{i}=x_{1}^{-4}$ & $x_{1}^{i}=x_{1}^{n+4}$ & $x_{1}^{i}=x_{1}^{-3} \div x_{1}^{n+3}$ & \\
$\left(\ell_{f}^{\alpha-1} \mathscr{B}\right)_{i}^{\prime}$ & $x_{1}^{i}=x_{1}^{-2}$ & $x_{1}^{i}=x_{1}^{n+2}$ & & $x_{1}^{i}=x_{1}^{-1} \div x_{1}^{n+1}$ \\
\hline
\end{tabular}

- with the central and forward schemes for the fourth order derivative of displacement at nodes $x_{1}^{-6} \div x_{1}^{-1}$ and for the third order derivative of strain at nodess $x_{1}^{-4} \div x_{1}^{1}$,

$$
\begin{array}{r}
\frac{u_{i-2}-4 u_{i-1}+6 u_{i}-4 u_{i+1}+u_{i+2}}{h^{4}}=\frac{u_{i}-4 u_{i+1}+6 u_{i+2}-4 u_{i+3}+u_{i+4}}{h^{4}}, \quad \text { for } x_{1}^{-6} \div x_{1}^{-1}, \\
\frac{-\grave{\gamma}_{i-2}+2 \grave{\gamma}_{i-1}-2 \grave{\gamma}_{i+1}+\grave{\gamma}_{i+2}}{2 h^{3}}=\frac{-\grave{\gamma}_{i}+3 \grave{\gamma}_{i+1}-3 \grave{\gamma}_{i+2}+\grave{\gamma}_{i+3}}{h^{3}}, \quad \text { for } x_{1}^{i}=x_{1}^{-4} \div x_{1}^{1} ;
\end{array}
$$

- with the central and backward schemes for the fourth order derivative of displacement at nodes $x_{1}^{n+1} \div x_{1}^{n+6}$ and for the third order derivative of strain at nodes $x_{1}^{n-1} \div x_{1}^{n+4}$

$$
\begin{array}{r}
\frac{u_{i-2}-4 u_{i-1}+6 u_{i}-4 u_{i+1}+u_{i+2}}{h^{4}}=\frac{u_{i-4}-4 u_{i-3}+6 u_{i-2}-4 u_{i-1}+u_{i}}{h^{4}}, \quad \text { for } x_{1}^{i}=x_{1}^{n+1} \div x_{1}^{n+6}, \\
\frac{-\grave{\gamma}_{i-2}+2 \grave{\gamma}_{i-1}-2 \grave{\gamma}_{i+1}+\grave{\gamma}_{i+2}}{2 h^{3}}=\frac{-\grave{\gamma}_{i-3}+3 \grave{\gamma}_{i-2}-3 \grave{\gamma}_{i-1}+\grave{\gamma}_{i}}{h^{3}}, \quad \text { for } x_{1}^{i}=x_{1}^{n-1} \div x_{1}^{n+4} .
\end{array}
$$

\subsection{Parametric study}

This section highlights the influence of the material parameters $\alpha$ and $\ell_{f}$ on the bending behaviour of the beam and compares the fractional and classical approaches to show the ability of taking into account the SE. A comparison of sFTB and s-FEBB is provided as well to emphasize the need of considering the shear effect when the beam is thick in relation to its length. The examples of beam with following data are thereby considered: beam length $L=100 \mu \mathrm{m}$, width $a=10 \mu \mathrm{m}$ and height $b=50 \mu \mathrm{m}$ of rectangular cross-section, homogenized Young's modulus $E=10 \mathrm{GPa}$, Poisson's ratio $v=0.2$ and $h=0.1 \mu \mathrm{m}$. The shear correction factor for rectangular cross-section is assumed $k=5 / 6$. In each of the examples, the beam was loaded with a concentrated force: at the mid-span for simply supported, fixed, and propped cantilever schemes, and at the free end for a cantilever scheme (see Fig. 2). The point load $P=10 \mu \mathrm{N}$ is introduced by equivalent continuous load [28,41]

$$
p_{3}(\xi)=\frac{k_{1} k_{2}}{2 \tanh \left(k_{1} / 2\right)} \frac{1}{\cosh ^{2}\left[k_{1}\left(\xi-L_{1}\right)\right]} \frac{P}{L},
$$

where $k_{1}=100$ and $k_{2}$ are dimensionless parameters, $k_{2}=\left(L_{1}+1\right)^{-200}+1$ for $L_{1} \in\langle 0.0 ; 0.5]$ and $k_{2}=$ $\left(-L_{1}+2\right)^{-200}+1$ for $L_{1} \in(0.5 ; 1.0], \xi=x_{1} / L$ is a dimensionless coordinate and $L_{1}$ is a point load position in relation to the beam length $\left(L_{1}=0.5\right.$ for load at the midpoint and $L_{1}=1.0$ for load at the end of beam). The boundary conditions are summarised in Table 2 . The effect of non-locality was investigated for the following parameters: $\alpha \in\{0.8,0.6\}$ and $\ell_{f}^{\max } \in\{0.001 L, 0.10 L, 0.2 L\}$ with a symmetric distribution that is smoothly decreasing at the boundaries (see Fig. 3), described by the following function [42] 
$\ell_{f}\left(x_{1}\right)= \begin{cases}\ell_{f}^{\max } \zeta_{1}^{3}\left(10-15 \zeta_{1}+6 \zeta_{1}^{2}\right) & \text { for } 0 \leq x_{1} \leq \eta_{1} \ell_{f}^{\max } \\ \ell_{f}^{\max } & \text { for } \eta_{1} \ell_{f}^{\max }<x_{1}<L-\eta_{2} \ell_{f}^{\max } \\ \ell_{f}^{\max } \zeta_{2}^{3}\left(10-15 \zeta_{2}+6 \zeta_{2}^{2}\right) & \text { for } L-\eta_{2} \ell_{f}^{\max } \leq x_{1} \leq L\end{cases}$

where $\zeta_{1}=x_{1} /\left(\eta_{1} \ell_{f}^{\max }\right), \zeta_{2}=\left(L-x_{1}\right) /\left(\eta_{2} \ell_{f}^{\max }\right)$ and for symmetric case $\eta_{1}=1.2, \eta_{2}=1.2$.

The results of this study, presented in Fig. 4, show the effect of a small scale on the deflection. It can be observed that parameters $\alpha$ and $\ell_{f}$ influence the stiffness of the beam. With a decrease in $\alpha$ or an increase in $\ell_{f}^{\max }$, the deflection of the simply supported and cantilever beam increases, while for propped cantilever and fixed beams the stiffening or softening effect is observed for certain values of these parameters. For length scale $\ell_{f}$, small in relation to $L\left(\ell_{f}^{\max }=0.001 L\right)$, the effect of non-locality disappears and the results are identical to the classic local formulation, as expected.

Moreover, in the above examples, the length to height ratio is $L / b=2$, and it is visible that for this beam geometry, the deflections predicted by s-FTB and s-FEBB differ significantly. Beam deflections predicted by s-FTB and sFEBB theories in the wider range of $L / b=2 \div 26$ are compared in Fig. 5. The maximum deflections are higher for s-FTB than the s-FEBB model, and similarly to the classic approach, the differences are noticeable for thick beams and negligible for slender beams. Moreover, the inclusion of the non-locality makes the shear effect more significant than in the classic approach. It is especially visible for the fixed beam scheme, where, for example, for the ratio $L / b=2$, the maximum deflection for the Timoshenko beam is about 3.85 times greater than for the Euler-Bernoulli beam in the classical approach $(\alpha=1)$ and 4.85 in fractional approach with $\alpha=0.6$ and $\ell_{f}^{\max }=0.1 \mathrm{~L}$. Therefore, the shear effect for small scale thick beam definitely should not be ignored and should even be considered for a higher ratio $L / b$ than in local theory. However, for a higher $L / b$ ratio, the ratio of deflections $\frac{u^{s-F T B}}{u^{s-F E B B}}$ reaches a value of 1.0. For this reason, for long beams, the s-FTB model can be reduced to the s-FEBB model without losing the correctness of the results - which is analogical to local Timoshenko and Euler-Bernoulli beams.

\section{Experimental validation}

To show the effectiveness of the developed model, it was validated based on the microcantilever bending experiment presented in [43]. The tested samples were made of a SU8 polymer with the following material parameters: the bulk value of Young's modulus $E_{b}=6.9 \mathrm{GPa}$ and Poisson's ratio $v=0.22$. These cantilevers had a length in the range of $L=80 \div 850 \mu \mathrm{m}$ and a rectangular cross-section with a width of $a=82 \mu \mathrm{m}$ and $a=122 \mu \mathrm{m}$, and a height of $b=8.4 \mu \mathrm{m}$ and $b=14.4 \mu \mathrm{m}$. These data allow for the analysis of non-local effects depending not only on the crosssection dimensions but also on the length of the beam. As shown in Fig. 8, decreasing the length of the beam causes a decrease in modulus of elasticity compared to the bulk value. Conversely, the decrease of the cross-section dimensions is manifested by the increase of Young's modulus. Moreover, we took this increase into account by analogy to the results obtained for iPP+0.2\% PACS polymer samples. Figure 6 shows the distribution of elastic modulus in cross-section measured for samples from the nanoindentation test, while Young's modulus from the tensile test is $1.75 \pm 0.1 \mathrm{GPa}$ (results derived from [44]). This map of Young's modulus indicates that it is not constant in the whole cross-section, but there is a core with other characteristics. Based on this information, we include the change of elastic modulus in $x_{3}$ direction by the core-shell (CS) model (see Fig. 7) with a stiffer core $\left(E_{c}=f E_{b}\right)$ of thickness $c$ and a bulk shell $\left(E_{b}\right)$, where $f$ means the ratio of the core Young's modulus $E_{s}$ and bulk Young's modulus $E_{b}$. The stiffness of the core-shell model can be expressed by the effective bending stiffness

$E_{e} I_{e}=E_{c} I_{c}+E_{b} I_{b}$

where $E_{e}$ is the effective Young's modulus and $I_{e}, I_{c}$ and $I_{b}$ are the moments of inertia of the effective (homogeneous) cross-section, the core, and the bulk shell respectively,

$I_{e}=\frac{a b^{3}}{12}, \quad I_{c}=\frac{a c^{3}}{12}, \quad I_{b}=\frac{a b^{3}-a c^{3}}{12}$.

Then, the effective Young's modulus is

$E_{e}=E_{b}\left(1+(f-1) \frac{c^{3}}{b^{3}}\right)$.

It was found that for SU-8 polymer rectangular samples the height of core zone is $c=7.0 \mu \mathrm{m}$, which has a Young modulus $f=1.91$ times higher than the bulk value, regardless of sample size. Subsequently, using the Eq. (41), the effective elastic modulus is 7.62 GPa for sample of dimensions $a=122 \mu \mathrm{m}, b=14.4 \mu \mathrm{m}$ and $10.52 \mathrm{GPa}$ for sample of dimensions $a=82 \mu \mathrm{m}, b=8.4 \mu \mathrm{m}$. Then, the parameters of s-FTB model were identified as $\alpha=0.75$ and $\ell_{f}^{\max }=5 \mu \mathrm{m}$ with asymmetric distribution (cf. Fig. 3) described by Eq. (38), where $\eta_{1}=1.2, \eta_{2}=2.0$. 
Fig. 2 Static schemes of the beams used in the parametric study under arbitrary transverse load: a simply supported, b fixed and $\mathbf{c}$ propped cantilever-with mid-span point load; and $\mathbf{d}$ cantilever-with end point load

Fig. 3 Assumed distributions of the length scale $\ell_{f}\left(x_{1}\right)$ along the beam length: symmetric case used in parametric study and asymmetric case used in validation [see also Eq. (38)] (a) simply supported beam

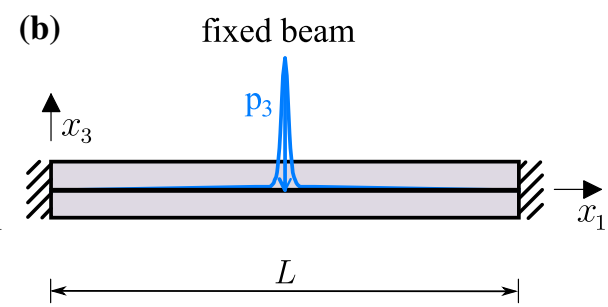

(c) propped cantilever beam

(d)

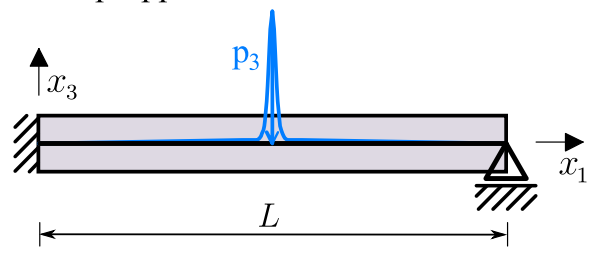

cantilever beam

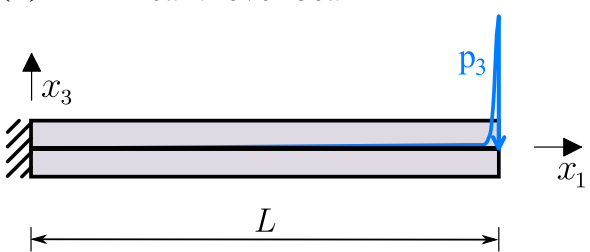

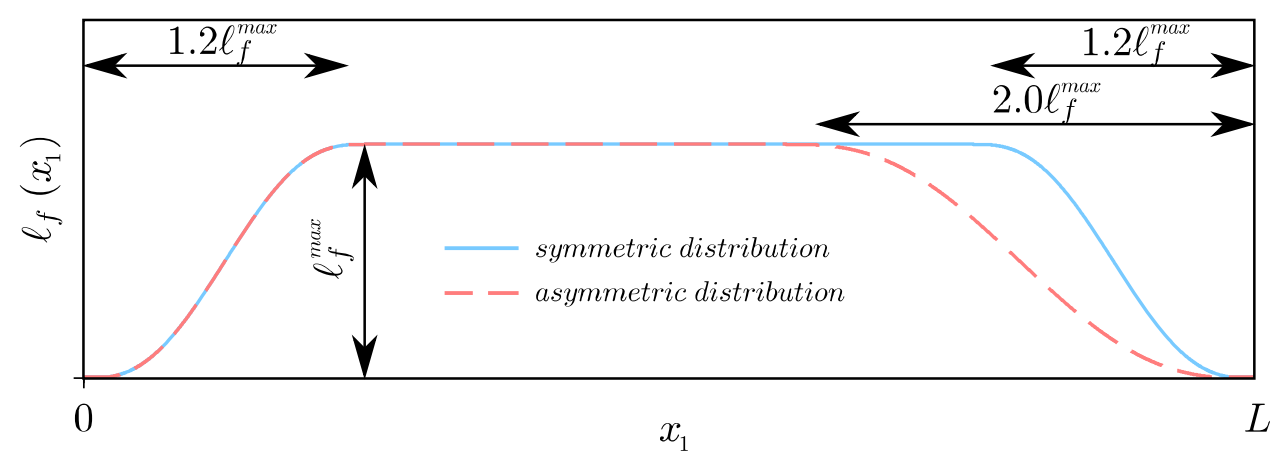

Table 2 Boundary conditions applied for the static schemes presented in Fig. 2 (see also Eq. (30) and Fig. 1)

\begin{tabular}{|c|c|c|}
\hline \multirow{2}{*}{$\frac{\text { Beam type }}{\text { Simply supported }}$} & \multicolumn{2}{|l|}{ Boundary conditions } \\
\hline & $\begin{array}{ll}\bar{u}_{3}\left(x_{1}^{0}\right)=0, & M_{2}\left(x_{1}^{0}\right)=0 \\
\bar{u}_{3}\left(x_{1}^{n}\right)=0, & M_{2}\left(x_{1}^{n}\right)=0\end{array}$ & $\begin{array}{ll}u_{0}=0, & -\mathcal{B}_{0}+\mathscr{A}_{0}=0 \\
u_{n}=0, & -\mathcal{B}_{n}+\mathscr{A}_{n}=0\end{array}$ \\
\hline Fixed & $\begin{array}{ll}\bar{u}_{3}\left(x_{1}^{0}\right)=0, & \Phi_{2}\left(x_{1}^{0}\right)=0 \\
\bar{u}_{3}\left(x_{1}^{n}\right)=0, & \Phi_{2}\left(x_{1}^{n}\right)=0\end{array}$ & $\begin{array}{l}u_{0}=0, \quad-\left(\ell_{f}^{\alpha-1}\right)_{0} \mathcal{A}_{0}+\left(-\mathcal{C}_{0}+\mathscr{B}_{0}\right) \frac{(E I)^{*}}{k(G A)^{*}}=0 \\
u_{n}=0, \quad-\left(\ell_{f}^{\alpha-1}\right)_{n} \mathcal{A}_{n}+\left(-\mathcal{C}_{n}+\mathscr{B}_{n}\right) \frac{(E I)^{*}}{k(G A)^{*}}=0\end{array}$ \\
\hline Propped cantilever & $\begin{array}{ll}\bar{u}_{3}\left(x_{1}^{0}\right)=0, & \Phi_{2}\left(x_{1}^{0}\right)=0 \\
\bar{u}_{3}\left(x_{1}^{n}\right)=0, & M_{2}\left(x_{1}^{n}\right)=0\end{array}$ & $\begin{array}{ll}u_{0}=0, & -\left(\ell_{f}^{\alpha-1}\right)_{0} \mathcal{A}_{0}+\left(-\mathcal{C}_{0}+\mathscr{B}_{0}\right) \frac{(E I)^{*}}{k(G A)^{*}}=0 \\
u_{n}=0, & -\mathcal{B}_{n}+\mathscr{A}_{n}=0\end{array}$ \\
\hline Cantilever & $\begin{array}{l}\bar{u}_{3}\left(x_{1}^{0}\right)=0, \quad \Phi_{2}\left(x_{1}^{0}\right)=0 \\
M_{2}\left(x_{1}^{n}\right)=0, \quad V_{3}\left(x_{1}^{n}\right)=0\end{array}$ & $\begin{array}{l}u_{0}=0, \quad-\left(\ell_{f}^{\alpha-1}\right)_{0} \mathcal{A}_{0}+\left(-\mathcal{C}_{0}+\mathscr{B}_{0}\right) \frac{(E I)^{*}}{k(G A)^{*}}=0 \\
-\mathcal{B}_{n}+\mathscr{A}_{n}=0, \quad-\mathcal{C}_{n}+\mathscr{B}_{n}=0\end{array}$ \\
\hline
\end{tabular}

The validation results are shown in Fig. 8, where the elastic modulus was calculated according to Timoshenko beam theory for a cantilever with a point load at the free end

$E=\left(\frac{F L^{3}}{3 I}+\frac{2(1+v) F L}{k A}\right) \frac{1}{\bar{u}_{3}}$.

It is observed, that the s-FTB model results agree very well with the measurement data. In addition, the results from the classical Timoshenko beam (CTB) model are plotted in Fig. 8, demonstrating that the classical approach is not suitable for describing the behavior of small scale beams.

\section{Conclusions}

In the presented work, the space-Fractional Timoshenko beam theory has been developed from space-Fractional Euler-Bernoulli beam theory by including the shear deformation. The effect of non-locality for bending of different beam types was studied and the results of the space-Fractional Timoshenko and space-Fractional Euler-Bernoulli beam theories were compared. The validation of the model was also provided. From these analyzes, the following conclusions are drawn: 
(a)

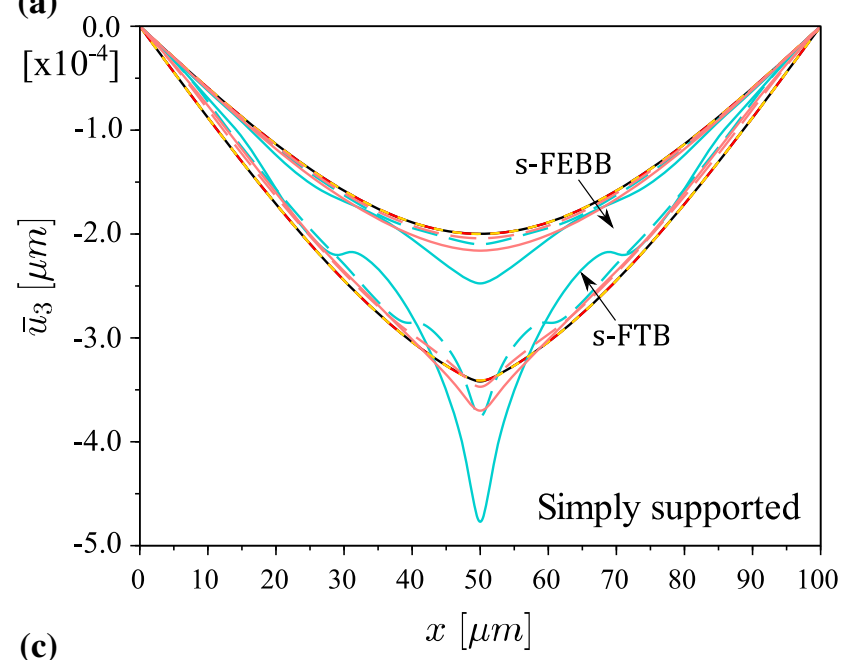

(c)

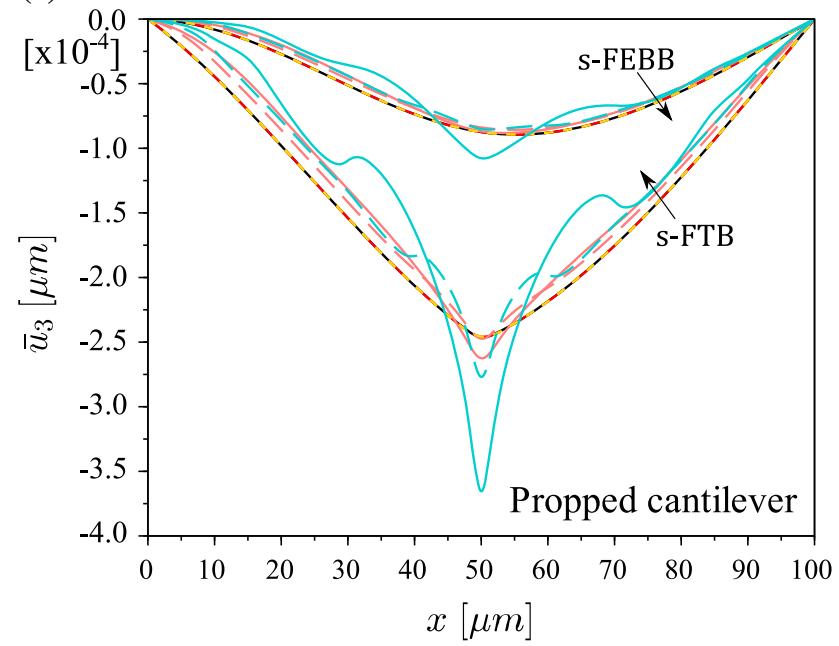

(b)
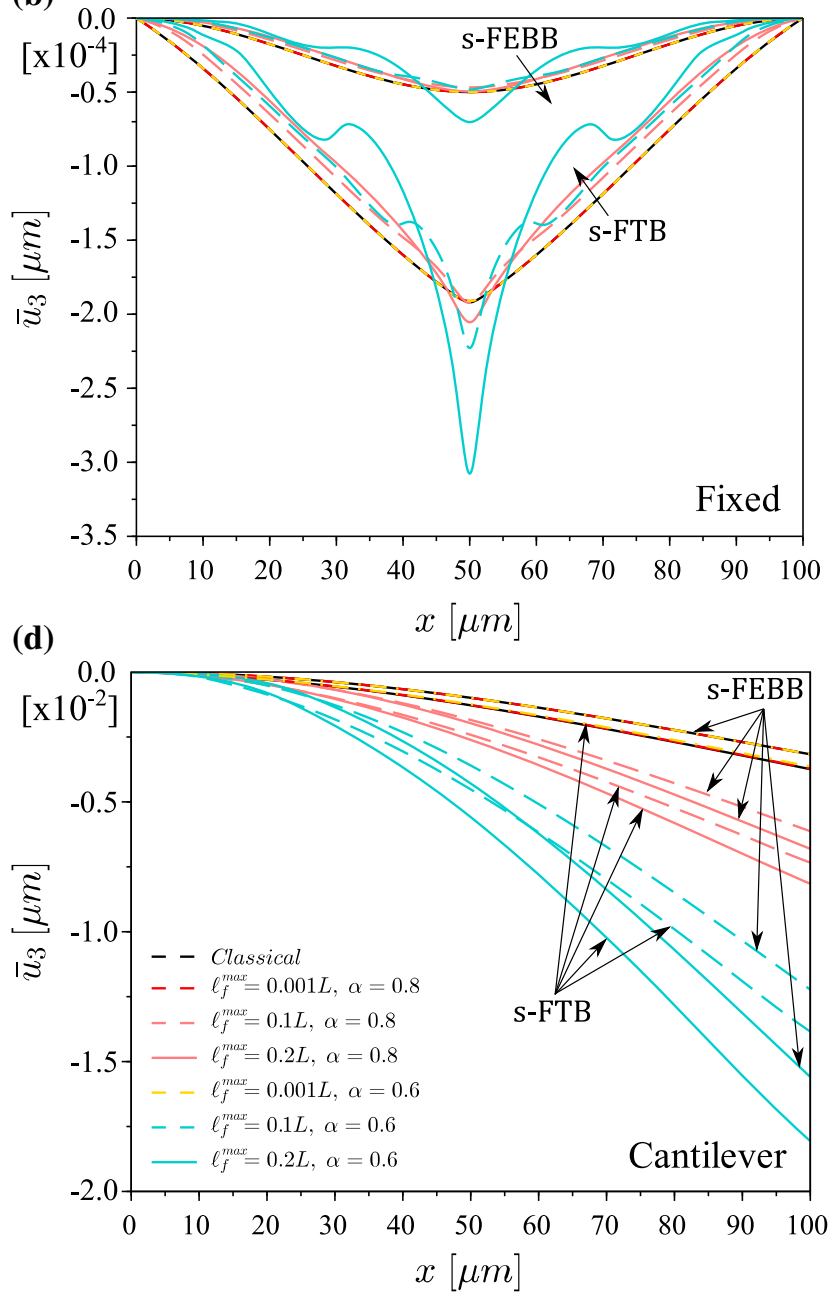

Fig. 4 Deflection of beam predicted by s-FTB and s-FEBB models for: a the simply supported, $\mathbf{b}$ the fixed and $\mathbf{c}$ the propped cantilever schemes with mid-span point load; and $\mathbf{d}$ the cantilever scheme with end point load, for $\alpha \in\{0.8,0.6\}, \ell_{f}^{\max } \in\{0.001 L, 0.10 L, 0.20 L\}$ and symmetric $\ell_{f}$ distribution (cf. Fig. 3)

- the numerical algorithm has been enriched with the shear effect while keeping the possibility of including any boundary conditions, any transverse load, and variable length scale,

- parameters $\alpha$ and $\ell_{f}$ control the stiffness of the fractional beam,

- the shear effect is more significant in non-local beams, therefore it should be considered even with more slender beams than in a local approach,

- the current model is adequate for modeling small scale short beams,

- the validation confirms the applicability of the presented model.

Acknowledgements This work is supported by the National Science Centre, Poland under Grant No. 2017/27/B/ST8/00351.
Open Access This article is licensed under a Creative Commons Attribution 4.0 International License, which permits use, sharing, adaptation, distribution and reproduction in any medium or format, as long as you give appropriate credit to the original author(s) and the source, provide a link to the Creative Commons licence, and indicate if changes were made. The images or other third party material in this article are included in the article's Creative Commons licence, unless indicated otherwise in a credit line to the material. If material is not included in the article's Creative Commons licence and your intended use is not permitted by statutory regulation or exceeds the permitted use, you will need to obtain permission directly from the copyright holder. To view a copy of this licence, visit http://creativecomm ons.org/licenses/by/4.0/.

\section{Appendix}

The equilibrium equations of fractional beam are derived from the principle of virtual work $\delta U=\delta W$, where $\delta U$ is the variation in the internal energy 
Table 3 Boundary conditions applied for the static schemes presented in Fig. 2

\begin{tabular}{|c|c|}
\hline Beam type & Boundary conditions \\
\hline Simply supported & 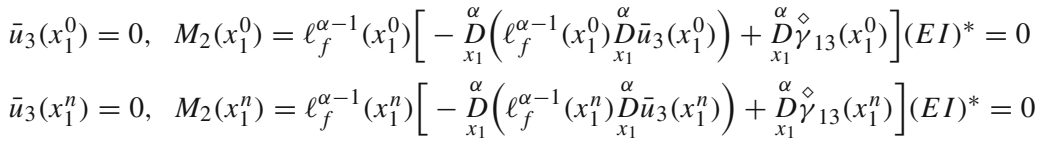 \\
\hline Fixed & $\begin{array}{l}\bar{u}_{3}\left(x_{1}^{0}\right)=0, \quad \Phi_{2}\left(x_{1}^{0}\right)=-\ell_{f}^{\alpha-1}\left(x_{1}^{0}\right) \stackrel{\alpha}{D_{1}} \bar{u}_{3}\left(x_{1}^{0}\right)+\stackrel{\diamond}{\gamma}_{13}\left(x_{1}^{0}\right)=0 \\
\bar{u}_{3}\left(x_{1}^{n}\right)=0, \quad \Phi_{2}\left(x_{1}^{n}\right)=-\ell_{f}^{\alpha-1}\left(x_{1}^{n}\right){\stackrel{\alpha}{D} \bar{u}_{1}}_{3}\left(x_{1}^{n}\right)+\stackrel{\diamond}{\gamma}_{13}\left(x_{1}^{n}\right)=0\end{array}$ \\
\hline Propped cantilever & $\begin{array}{l}\bar{u}_{3}\left(x_{1}^{0}\right)=0, \quad \Phi_{2}\left(x_{1}^{0}\right)=-\ell_{f}^{\alpha-1}\left(x_{1}^{0}\right) \stackrel{\alpha}{D_{1}} \bar{u}_{3}\left(x_{1}^{0}\right)+\stackrel{\stackrel{\gamma}{\gamma}}{13}_{13}\left(x_{1}^{0}\right)=0, \\
\bar{u}_{3}\left(x_{1}^{n}\right)=0, \quad M_{2}\left(x_{1}^{n}\right)=\ell_{f}^{\alpha-1}\left(x_{1}^{n}\right)\left[-\stackrel{\alpha}{D}\left(\ell_{f}^{\alpha-1}\left(x_{1}^{n}\right) \underset{x_{1}}{D} \bar{u}_{3}\left(x_{1}^{n}\right)\right)+{\stackrel{\alpha}{D} \stackrel{\diamond}{\gamma}_{13}}_{x_{1}}\left(x_{1}^{n}\right)\right](E I)^{*}=0\end{array}$ \\
\hline Cantilever & 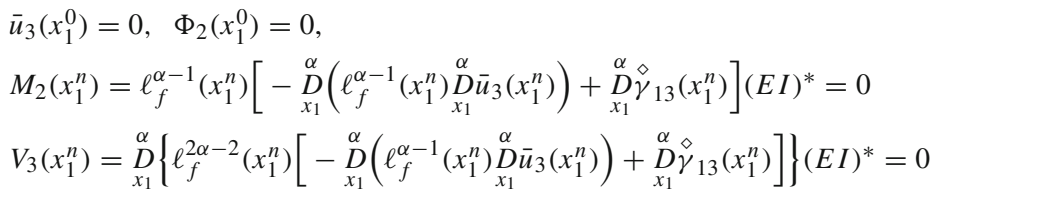 \\
\hline
\end{tabular}

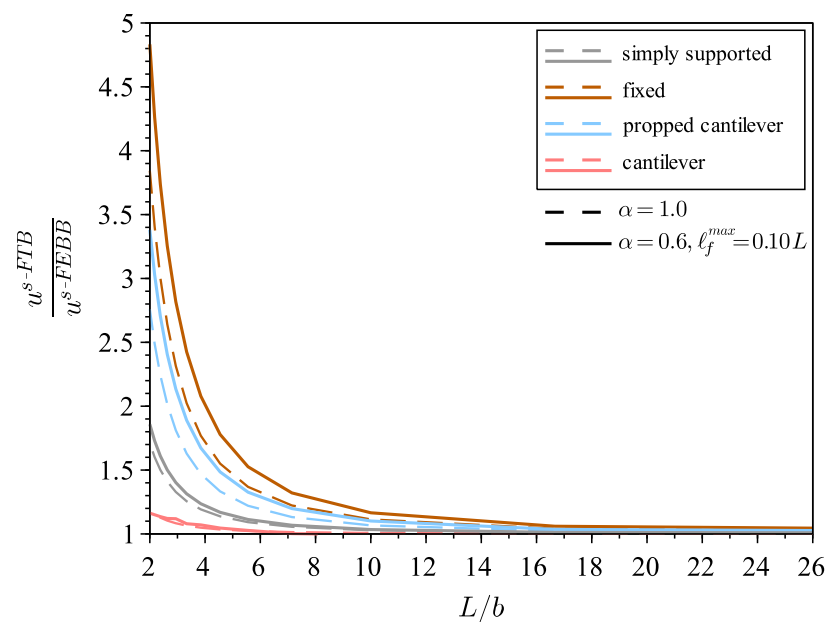

Fig. 5 Comparison of maximum deflection for s-FTB and s-FEBB models for: the simply supported scheme, the fixed scheme and the propped cantilever scheme with mid-span point load and the cantilever scheme with end point load, for $\alpha=0.6, \ell_{f}^{\max }=0.10 \mathrm{~L}$ and symmetric $\ell_{f}$ distribution

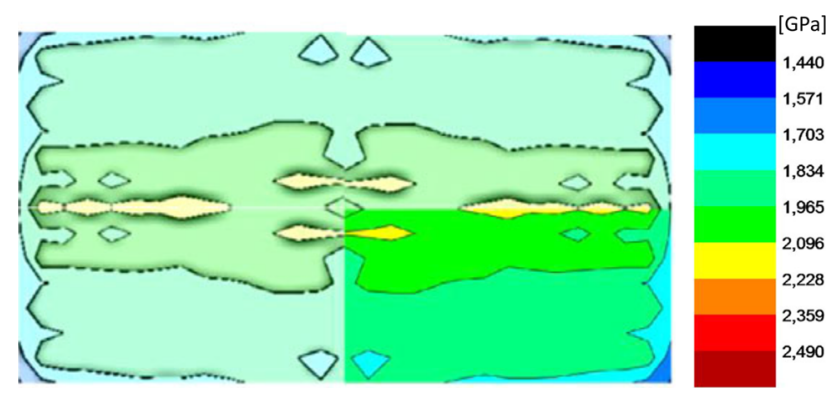

Fig. 6 Local elasticity modulus map from nanoindentation test for polymer (iPP+0.2\% PACS) sample, reprinted from [44] with permission of John Wiley and Sons

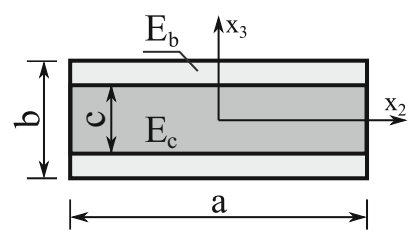

Fig. 7 Core-shell model of rectangular sample consisting of: the stiffer core of a Young's modulus $E_{c}$ and thickness of $c$; shell of a bulk Young's modulus $E_{b}$

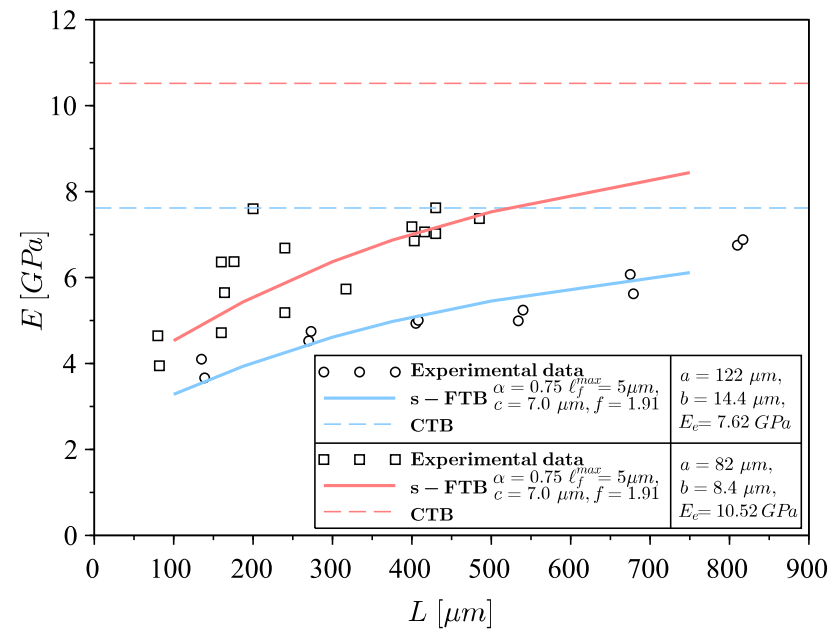

Fig. 8 The comparison of experimental measurements [43] (elastic modulus) of SU-8 polymer microcantilevers loaded at the free end, vs. the results of s-FTB model and CTB model 


$$
\begin{aligned}
\delta U & =\int_{V} \delta \boldsymbol{\varepsilon}^{T} \sigma \mathrm{d} V=\int_{L} \int_{A}\left(\delta \stackrel{\diamond}{\varepsilon}_{11} \sigma_{11}+2 \delta \stackrel{\diamond}{\varepsilon}_{13} \sigma_{13}\right) \mathrm{d} A \mathrm{~d} L= \\
& =\int_{L} \int_{A}\left[x_{3} \ell_{f}^{\alpha-1} \sigma_{11} \underset{x_{1}}{D} \delta \Phi_{2}+\left(\delta \Phi_{2} \underset{x_{3}}{D} x_{3}+\underset{x_{1}}{D} \delta \bar{u}_{3}\right) \ell_{f}^{\alpha-1} \sigma_{13}\right] \mathrm{d} A \mathrm{~d} L
\end{aligned}
$$

and introducing Eqs. (10), (11) and $\ell_{f}^{\alpha-1}{ }_{x_{3}}^{D} x_{3}=1$,

$\delta U=\int_{L}\left[M_{2} \ell_{f}^{\alpha-1} \underset{x_{1}}{D} \delta \Phi_{2}+\left(\delta \Phi_{2}+\ell_{f}^{\alpha-1} \underset{x_{1}}{D} \delta \bar{u}_{3}\right) V_{3}\right] \mathrm{d} L$

and $\delta W$ is the variation in the external work

$\delta W=\int_{L} p_{3} \delta \bar{u}_{3} \mathrm{~d} L$

To obtain the equilibrium equations, we look for a minimum of the functional $\mathcal{J}$

$$
\begin{aligned}
& \delta \mathcal{J}=\delta U-\delta W \\
& =\int_{L}\left[\left(M_{2} \ell_{f}^{\alpha-1} \underset{x_{1}}{D} \delta \Phi_{2}+\delta \Phi_{2} V_{3}\right)+\left(\ell_{f}^{\alpha-1} V_{3} \underset{x_{1}}{D} \delta \bar{u}_{3}-p_{3} \delta \bar{u}_{3}\right)\right] \mathrm{d} L=0,
\end{aligned}
$$

which, using the fractional Euler-Lagrange equation $[45,46]$, provides

$$
\begin{aligned}
& V_{3}-\stackrel{\alpha}{\underset{x_{1}}{D}}\left(M_{2} \ell_{f}^{\alpha-1}\right)=0 \rightarrow V_{3}=\stackrel{\alpha}{\underset{x_{1}}{D}}\left(M_{2} \ell_{f}^{\alpha-1}\right), \\
& p_{3}+\stackrel{\alpha}{D}\left(V_{x_{1}} \ell_{f}^{\alpha-1}\right)=0 \rightarrow p_{3}=-\stackrel{\alpha}{D}_{x_{1}}\left(V_{3} \ell_{f}^{\alpha-1}\right) .
\end{aligned}
$$

The boundary conditions for selected beam types are presented in Table 3.

\section{References}

1. Williams E (1957) Some observations of leonardo, galileo, mariotte and others relative to size effect. Ann Sci 13:23-29

2. Voyiadjis GZ, Yaghoobi M (2019) Size effects in plasticity. Academic Press, New York

3. Colas G, Serles P, Saulot A, Filleter T (2019) Strength measurement and rupture mechanisms of a micron thick nanocrystalline MOS 2 coating using AFM based micro-bending tests. J Mech Phys Solids 128:151-161

4. Fleck N, Muller G, Ashby M, Hutchinson J (1994) Strain gradient plasticity: theory and experiment. Acta Metall Mater 42:475-487

5. Stolken J (1997) The role of oxygen in nickel-sapphire interface fracture. Ph.D. dissertation, University of California, Santa Barbara

6. Hassanpour S, Heppler GR (2017) Micropolar elasticity theory: a survey of linear isotropic equations, representative notations, and experimental investigations. Math Mech Solids 22:224-242

7. Schijve J (1966) Note on couple stresses. J Mech Phys Solids 14:113-120

8. Marzec I, Boińbski J (2019) On some problems in determining tensile parameters of concrete model from size effect tests. Polish Maritime Res 26:115-125
9. Kumar R, Rani R, Miglani A (2019) A problem of axisymmetric vibration of nonlocal microstretch thermoelastic circular plate with thermomechanical sources. J Solid Mech 11:1-13

10. Pavlovic I, Pavlovic R, Janevsky G (2019) Mathematical modeling and stochastic stability analysis of viscoelastic nanobeams using higher-order nonlocal strain gradient theory. Arch Mech 71:137153

11. Challamel N (2018) Static and dynamic behaviour of nonlocal elastic bar using integral strain-based and peridynamic models. Comptes Rendus - Mecanique 346:320-335

12. Drapaca C, Sivaloganathan S (2019) Brief review of continuum mechanics theories. Fields Inst Monogr 37:5-37

13. Patnaik S, Sidhardh S, Semperlotti F (2021) Towards a unified approach to nonlocal elasticity via fractional-order mechanics. Int J Mech Sci 189:105992

14. Patnaik S, Hollkamp J, Semperlotti F (2020) Applications of variable-order fractional operators: A review. Proc R Soc A: Math Phys Eng Sci 476**

15. Barretta R, Faghidian S, Marotti de Sciarra F, Vaccaro M (2020) Nonlocal strain gradient torsion of elastic beams: variational formulation and constitutive boundary conditions. Arch Appl Mech 90:691-706

16. Lam JK, Koay SC, Lim CH, Cheah KH (2019) A voice coil based electromagnetic system for calibration of a sub-micronewton torsional thrust stand. Measurement 131:597-604

17. Dang V-H, Nguyen D-A, Le M-Q, Duong T-H (2020) Nonlinear vibration of nanobeams under electrostatic force based on the nonlocal strain gradient theory. Int J Mech Mater Des 16:289-308

18. Chandraseker K, Mukherjee S, Paci J, Schatz G (2009) An atomistic-continuum cosserat rod model of carbon nanotubes. $\mathrm{J}$ Mech Phys Solids 57:932-958

19. Marsden J, Hughes T (1983) Mathematical foundations of elasticity. Prentice-Hall, New Jersey

20. Holzapfel G (2000) Nonlinear solid mechanics-a continuum approach for engineering. Wiley, Hoboken

21. Haupt $P$ (2002) Continuum mechanics and theory of materials, 2nd edn. Springer, Berlin

22. Michelitsch T, Collet B, Riascos A, Nowakowski A, Nicolleau F (2016) A fractional generalization of the classical lattice dynamics approach. Chaos, Solitons Fractals 92:43-50

23. Postek E, Pecherski R, Nowak Z (2019) Peridynamic simulation of crushing processes in copper open-cell foam. Arch Metall Mater 64:1603-1610

24. Romano G, Barretta R (2017) Stress-driven versus strain-driven nonlocal integral model for elastic nano-beams. Compos B Eng 114:184-188

25. Babu B, Patel BP (2019) On the finite element formulation for second-order strain gradient nonlocal beam theories. Mech Adv Mater Struct 26:1316-1332

26. Failla G, Santini A, Zingales M (2013) A non-local twodimensional foundation model. Arch Appl Mech 83:253-272

27. Sumelka W, Blaszczyk T, Liebold C (2015) Fractional eulerbernoulli beams: theory, numerical study and experimental validation. Eur J Mech A Solids 54:243-251. https://doi.org/10.1016/ j.euromechsol.2015.07.002

28. Stempin P, Sumelka W (2020) Space-fractional euler-bernoulli beam model-theory and identification for silver nanobeam bending. Int J Mech Sci 186

29. Groves J, Wadley H (1997) Functionally graded materials synthesis via low vacuum directed vapor deposition. Compos B Eng 28:5769

30. Moon J, Caballero A, Hozer L, Chiang Y-T, Cima M (2001) Fabrication of functionally graded reaction infiltrated sic-si composite by three-dimensional printing ( $3 \mathrm{dptm})$ process. Mater Sci Eng, A 298:110-119 
31. Shen H, Wen J, Yu D, Wen X (2015) Stability of clamped-clamped periodic functionally graded material shells conveying fluid. JVC/J Vib Control 21:3034-3046

32. Swaminathan K, Sangeetha D (2017) Thermal analysis of FGM plates-a critical review of various modeling techniques and solution methods. Compos Struct 160:43-60

33. Sumelka W (2014) Thermoelasticity in the framework of the fractional continuum mechanics. J Therm Stresses 37:678-706

34. Sumelka W, Blaszczyk T (2014) Fractional continua for linear elasticity. Arch Mech 66:147-172

35. Podlubny I (1999) Fractional differential equations, volume 198 of mathematics in science and engineering. Academin Press

36. Sumelka W (2017) On fractional non-local bodies with variable length scale. Mech Res Commun 86:5-10

37. Szajek K, Sumelka W (2019) Discrete mass-spring structure identification in nonlocal continuum space-fractional model. Eur Phys J Plus 134:448

38. Szajek K, Sumelka W, Blaszczyk T, Bekus K (2020) On selected aspects of space-fractional continuum mechanics model approximation. Int J Mech Sci 167

39. Leszczyński J (2011) An introduction to fractional mechanics. Monographs No 198, The Publishing Office of Czestochowa University of Technology

40. Odibat Z (2006) Approximations of fractional integrals and Caputo fractional derivatives. Appl Math Comput 178:527-533

41. Magnucki K, Lewiński J (2019) Bending of beams with symmetrically varying mechanical properties under generalized loadshear effect. Eng Trans 67:441-457. https://doi.org/10.24423/ ENGTRANS.987.20190509

42. Abaqus (2012) Abaqus version 6.12 collection. SIMULIA Worldwide Headquarters, Providence, RI

43. Liebold C (2015). Größeneffekt in der elastizität. https://doi.org/ 10.14279/DEPOSITONCE-4967

44. Nowicki M (2018) Nanomechanical analysis of nucleation modified isotactic polypropylene. Macromol Symp 378:1600175. https://doi.org/10.1002/masy.201600175
45. Odzijewicz T, Malinowska AB, Torres DF (2012) Fractional variational calculus with classical and combined caputo derivatives. Nonlinear Anal Theory Methods Appl 75:1507-1515. https://doi. org/10.1016/j.na.2011.01.010

46. Malinowska A, Torres D (2011) Fractional calculus of variations for a combined caputo derivative. Fract Calc Appl Anal. https:// doi.org/10.2478/s13540-011-0032-6

Publisher's Note Springer Nature remains neutral with regard to jurisdictional claims in published maps and institutional affiliations. 